\title{
Anti-reflux medication use in preterm infants
}

\author{
Haslina Binti Abdul Hamid ${ }^{1,2}$, Lisa Szatkowski ${ }^{1}$, Helen Budge ${ }^{1}$ and Shalini Ojha ${ }^{1,3} \bowtie$ \\ (c) The Author(s) 2021
}

BACKGROUND: Current recommendations do not support the use of anti-reflux medications to treat gastro-oesophageal reflux disease (GORD) among preterm infants.

OBJECTIVE: To describe the prevalence of GORD and the use of anti-reflux medications amongst very preterm infants $(<32$ weeks' gestational age (GA)) in neonatal units in England and Wales.

DESIGN: Retrospective cohort study using the National Neonatal Research Database.

RESULTS: Among 58,108 infants [median GA (IQR) 29 (27-30) weeks], 15.8\% ( $n=9191)$ had a diagnosis of GORD and 36.9\% $(n=12,446)$ received anti-reflux medications. Those who received anti-reflux medications were more preterm [GA, median (IQR): medications, 28 (26-30) vs. no medications, 30 (28-31); $p<0.001]$ and had lower birth weight [mean (SD): medications, $1124 \mathrm{~g}(354)$ vs. no medications, $1265 \mathrm{~g}$ (384); $p<0.001]$. Most $(57 \%, n=12,224)$ received Gaviscon, or Histamine-2 Receptor Antagonist (H2RA) $(56 \%, n=11,959)$. Over time, prokinetic use has declined substantially, the use of H2RAs and Gaviscon has reduced although they continue to be used frequently, whilst the use of PPIs has increased.

CONCLUSIONS: Anti-reflux medications are frequently prescribed in very preterm infants, despite evidence to suggest that they are not effective and may be harmful. Clear guidelines for diagnosing GORD and the use of anti-reflux medications are required to rationalise the pharmacological management of GORD in preterm infants.

Pediatric Research (2022) 92:520-525; https://doi.org/10.1038/s41390-021-01821-y

\section{IMPACT:}

- Anti-reflux medications are frequently prescribed, often without a diagnosis of gastro-oesophageal reflux disease, to very preterm infants while in the neonatal unit and at discharge.

- Half of the infants born at $<28$ weeks' gestational age receive anti-reflux medications in hospital and a quarter are discharged home on them.

- Although the use of prokinetics declined following alerts of adverse events, histamine2-receptor antagonists and alginates such as Gaviscon continue to be used and the use of proton-pump inhibitors has increased more than 2-fold.

\section{INTRODUCTION}

Gastro-oesophageal reflux (GOR), the passage of gastric contents into the oesophagus, is common in preterm infants. ${ }^{1}$ It usually occurs due to the relatively abundant volume of liquid intake and supine feeding position. However, GOR can worsen and lead to troublesome signs and symptoms i.e., gastro-oesophageal reflux disease (GORD).

Determination of the exact prevalence of GOR versus GORD is challenging because of an unclear distinction between physiologic and pathologic reflux. Additionally, the terms "reflux", "acidreflux", and GORD are often used interchangeably by healthcare professionals as well parents and families of infants. ${ }^{2}$ In a retrospective cohort study of 33 neonatal units in the USA, ${ }^{3}$ there was a wide variation between the units in the proportion of preterm infants who received a diagnosis of GORD based on the International Classification of Diseases (ICD-9). Approximately $10 \%$ of infants born at 22-36 weeks gestational age (GA) were recorded to have GORD with diagnosis prevalence ranging from 2.4 to $29.9 \%$ across the included neonatal units.

Anti-reflux medications, including Histamine-2 receptor antagonists (H2RA) and proton-pump inhibitors (PPI), are unlicensed for use in neonates in the UK and many other countries, ${ }^{4}$ though offlabel use is frequently reported. ${ }^{5}$ Antacids and other acidsuppressing agents may reduce gastric acidity but anti-reflux medications do not improve signs/symptoms of GORD and their use has been associated with increased risks of adverse outcomes including necrotising enterocolitis (NEC) and infections. ${ }^{6}$ The North American Society for Pediatric Gastroenterology, Hepatology, and Nutrition and European Society for Paediatric Gastroenterology Hepatology and Nutrition (NASPGHAN and ESPGHAN combined) clinical practice guidelines ${ }^{1}$ suggests nonpharmacological approaches to GORD before pharmacological therapy. Similarly, the American Academy of Paediatrics recommends that anti-reflux medications should be used with caution, if

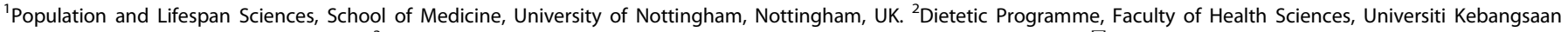
Malaysia, 50300 Kuala Lumpur, Malaysia. ${ }^{3}$ Neonatal Unit, University Hospitals of Derby and Burton, Derby DE23 3DT, UK. ${ }^{\mathrm{em}}$ ail: shalini.ojha@nottingham.ac.uk

Received: 29 July 2021 Revised: 28 September 2021 Accepted: 1 October 2021

Published online: 29 October 2021 
at all, in preterm infants due to lack of evidence of efficacy and possible significant harm. ${ }^{7}$

Despite these recommendations, anti-reflux medications are frequently used. A large retrospective study including infants admitted to neonatal units in 43 children's hospitals in the USA reported that $24 \%$ received either an H2RA or PPI with the extremely preterm infants being the most likely to receive these medicines. ${ }^{8}$ Similarly, Malcolm et al. ${ }^{9}$ reported that a quarter of extremely low birth weight infants who were enrolled in the National Institute of Child Health and Human Development Neonatal Network generic database in 2002-2003 were discharged from the hospital with anti-reflux medications.

In the UK, two survey studies of neonatal healthcare professionals, in $2004^{10}$ and $2018^{11}$ revealed that clinicians reported frequently prescribing anti-reflux medications in neonatal units. Dhillon and Ewer ${ }^{10}$ reported that, in 2004, nearly all respondents used anti-reflux medications to manage GOR. In 2018, another survey showed that the use of anti-reflux medications remained popular ${ }^{11}$ despite the increasing evidence of lack of efficacy and possible harm. Both studies analysed the use of medications as reported by clinicians. There are no studies that have analysed the prevalence of GORD diagnosis and the actual use of anti-reflux medications in neonatal units in the UK.

In this study, we aimed to describe the prevalence of GORD diagnosis and use of anti-reflux medications amongst very preterm infants in England and Wales. We also aimed to analyse the types of medications used, duration and trend of medications use between 2010 and 2017.

\section{METHODS}

We conducted a retrospective observational cohort study using the data held in the National Neonatal Research Database (NNRD). The NNRD is a repository of clinical data of admissions to the 200 neonatal units in England, Wales and Scotland that make up the UK Neonatal Collaborative. ${ }^{12}$

\section{Identification of study cohort}

Infants born $<32$ weeks' gestational age (GA), in England and Wales from 2010 until 2017, whose data are held in the NNRD were included. Infants were excluded if they were missing data on sex, birth weight, month of birth or one or more days of care, were a late admission to neonatal care $(>24 \mathrm{~h}$ ) or had an extreme birth weight for GA (Z-score $\geq+4$ SD or $\leq-4 S D)$.

\section{Data extraction}

Records of GORD were extracted by searching for specified terms in the "principal diagnosis at admission", "daily diagnoses" and "diagnoses at discharge" fields. A list of anti-reflux medications for GORD was prepared according to the British National Formulary (BNF) and included Ranitidine (H2RA), Omeprazole and Lansoprazole (PPI), Domperidone, Metoclopramide, and Erythromycin (Prokinetics). Indicator variables were created for each day of care to show whether an infant was prescribed any of the antireflux medications. Code lists are available from the authors on request.

\section{Data analysis}

Statistical analysis was performed using STATA 16.0 software (Stata Corp. College Station, TX). Descriptive statistics were used to describe infant characteristics, the prevalence of GORD and the use of anti-reflux medications. Values are presented as numbers and percentages for categorical data and for continuous variables, mean [ \pm standard deviation (SD)] used for normally distributed data and median [interquartile range (IQR)] for non-normally distributed data. Linear regression was used where appropriate to quantify trends over time in medication use.

\section{RESULTS}

Among the total of 58,108 infants, $69.9 \%(n=40,607)$ were born at 28-31 weeks GA and $30.1 \%(n=17,501)$ were born at $<28$ weeks GA. $45.8 \%(n=26,596)$ were female. Median (IQR) GA was 29 weeks (27-30), and the mean (SD) birth weight was $1213 \mathrm{~g}$ (379). The median (IQR) length of hospital stay was 49 days (34-74) and median (IQR) postmenstrual age (PMA) at discharge was 36 weeks (35-38).

Table 1 shows that $15.8 \%(n=9191)$ of total infants had any record of GORD. Infants first received GORD diagnosis at median (IQR) PMA of 36 (33-38) weeks. Infants with a diagnosis of GORD were more preterm than infants without a diagnosis [GA, median (IQR): GORD, 28 weeks (26-30) vs. no GORD, 29 weeks (27-31); $p<0.001$ ], had lower birth weight [mean (SD): GORD, $1114 \mathrm{~g}$ (337) vs. no GORD, $1232 \mathrm{~g}(384) ; p<0.001$ ] and fewer were female [ $n$ (\%): GORD, 3856 (42.0\%) vs. no GORD, 22,740 (46.5\%); $p<0.001]$.

Of all the infants, $36.9 \%(n=21,446)$ received anti-reflux medications during admission and $18.2 \%$ were receiving them at discharge (Table 1). However, among those who received antireflux medications, only $39.6 \%$ had a diagnosis of GORD. The PMA [median (IQR)] at first prescription of an anti-reflux medication was 32 weeks (30-34) and the treatments were continued for a median (IQR) duration of 20 days (9-37). 49.3\% $(n=8634 / 17,501)$ of extremely preterm infants received anti-reflux medications during admission and $24.9 \%(n=4352 / 17,501)$ were receiving them at discharge. Infants who received anti-reflux medications were more preterm [GA, median (IQR): medications, 28 (26-30) vs. no medications, 30 (28-31); $p<0.001$ ], had lower birth weight [mean (SD): medications, $1124 \mathrm{~g}$ (354) vs. no medications, $1265 \mathrm{~g}$ (384); $p<0.001]$ and fewer were female $[n(\%)$ : medications, 9172 $(42.8 \%)$ vs. no medications, $17,424(47.5 \%) ; p<0.001]$.

Table 2 shows that among all infants who received anti-reflux medications $(36.9 \%, n=21,466)$, the largest number had Gaviscon $(57 \%, n=12,224)$ followed by $\operatorname{H2RA}(55.8 \%, n=11,959)$, prokinetics $(45.4 \%, n=9734)$ and PPI $(16.2 \%, n=3480)$. The use of these medications among the subgroups of $<28$ weeks' and 28-32 weeks' GA is given in Table 2. Extremely preterm infants received all types of medications more frequently and for longer.

During their stay, $11.4 \%(n=6624)$ infants received two and $6.4 \%(n=3687)$ received three types of anti-reflux medications. $16.7 \%(n=9694)$ of infants received two or more anti-reflux

Table 1. Diagnosis of gastro-oesophageal reflux disease (GORD) and use of anti-reflux medications in infants born at $<32$ weeks' gestational age in England and Wales from 2010 to 2017

\begin{tabular}{|c|c|c|c|}
\hline & $\begin{array}{l}\text { All infants } \\
n=58,108\end{array}$ & $\begin{array}{l}<28 \text { weeks' gestation } \\
n=17,501\end{array}$ & $\begin{array}{l}\text { 28-31 weeks' gestation } \\
n=40,607\end{array}$ \\
\hline PMA (weeks) at first GORD diagnosis, median (IQR) & $36(33-38)$ & $36(32-39)$ & $35(33-37)$ \\
\hline PMA (weeks) at first anti-reflux prescription, median (IQR) & $32(30-34)$ & $30(28-34)$ & $32(31-34)$ \\
\hline Number of days of anti-reflux medications, median (IQR) & $20(9-37)$ & $27(11-49)$ & $18(9-30)$ \\
\hline
\end{tabular}

GORD gastro-oesophageal disease, PMA postmenstrual age, IQR interquartile range. 
Table 2. Types of anti-reflux medication and number of days prescribed in infants born at $<32$ weeks' gestational age in England and Wales from 2010 to 2017.

\begin{tabular}{|llll}
\hline Received anti-reflux medications & & & \\
\hline Types of medications and number of days prescribed & $\begin{array}{l}\text { Total infants } \\
\boldsymbol{n}=\mathbf{2 1 , 4 6 6}\end{array}$ & $\begin{array}{l}<\mathbf{2 8} \text { weeks' gestation } \\
\boldsymbol{n}=\mathbf{8 6 3 4}\end{array}$ & $\begin{array}{l}\mathbf{2 8 - 3 1} \text { weeks' gestation } \\
\boldsymbol{n}=\mathbf{1 2 , 8 1 2}\end{array}$ \\
\hline Gaviscon, $n$ (\%) & $12,224(57.0)$ & $4467(51.7)$ & $7757(60.5)$ \\
\hline Number of days, median (IQR) & $17(8-29)$ & $21(10-37)$ & $15(8-25)$ \\
\hline PPI, $n$ (\%) & $3480(16.2)$ & $1736(20.1)$ & $1744(13.6)$ \\
\hline Number of days, median (IQR) & $20(10-34)$ & $24(11-41)$ & $18(9-29)$ \\
\hline H2RA, $n$ (\%) & $11,959(55.8)$ & $5533(64.1)$ & $6426(50.2)$ \\
\hline Number of days, median (IQR) & $15(6-30)$ & $17(7-35)$ & $14(6-26)$ \\
\hline Prokinetics, $n$ (\%) & $9734(45.4)$ & $4231(49.0)$ & $5503(43.0)$ \\
\hline Number of days, median (IQR) & $21(10-37)$ & $27(12-48)$ & $19(9-31)$
\end{tabular}

PPI proton-pump inhibitor, $H 2 R A H 2$ receptor antagonists, IQR interquartile range.

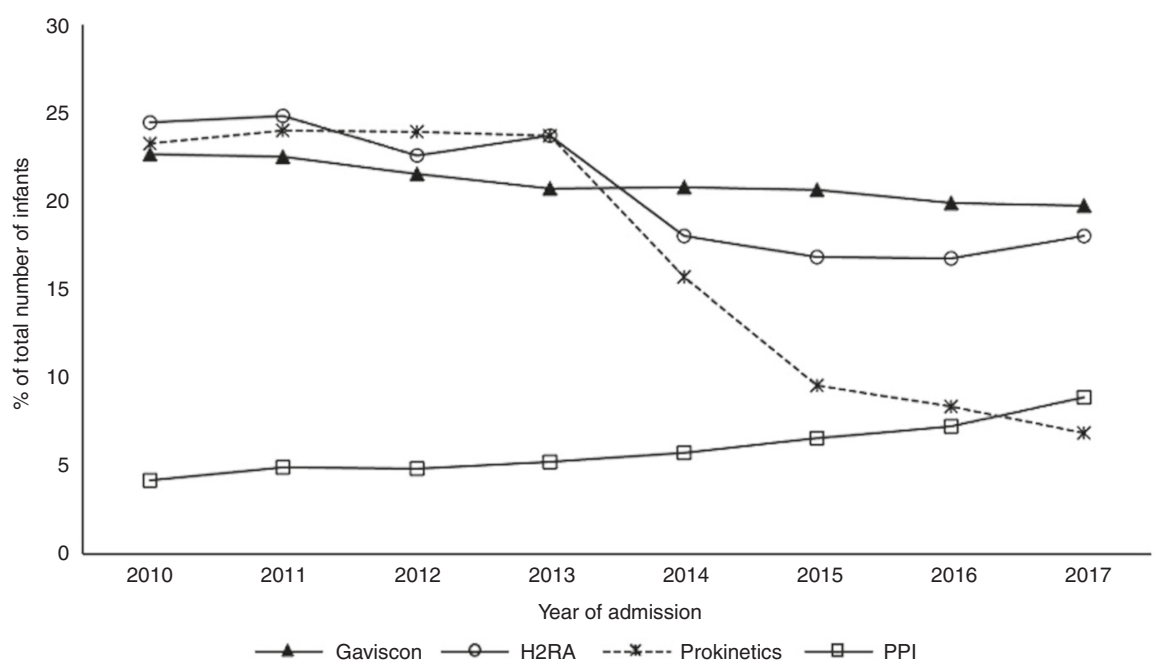

Fig. 1 Trends in prescribing anti-reflux medications among infants born at <32 weeks' gestational age in England and Wales from 2010 to 2017. H2RA histamine-2 receptor antagonists, PPI proton-pump inhibitors.

medications on the same day of care and $8.6 \%(n=4986)$ of infants were on two or more anti-reflux medications at discharge.

The change in prescribing of anti-reflux medications between 2010 and 2017 is shown in Fig. 1. The use of prokinetics sharply declined after 2013 (from 23.7\% in 2013 to 6.9\% in 2017). There was a smaller reduction in the use of H2RAs after 2013 (from $23.7 \%$ in 2013 to $17.9 \%$ in 2017). There was a linearly decreasing trend in the use of Gaviscon over the time period (from $20.7 \%$ in 2013 to $19.7 \%$ in $2017, \beta=-0.44,95 \% \mathrm{Cl}-0.55$ to $-0.32, p<$ $0.001)$ ). The proportion of infants prescribed with PPI increased more than twofold $(4.2 \%$ in 2010 to $8.9 \%$ in $2017, \beta=0.60,95 \% \mathrm{Cl}$ 0.40 to $0.79, p<0.001)$.

\section{DISCUSSION}

Anti-reflux medications were prescribed to $37 \%$ of infants born at $<32$ weeks' GA in England and Wales and over 18\% were discharged home on these medicines. We found that, on average, anti-reflux medications are started at 32 weeks' PMA and given for 3 weeks. Our findings show that anti-reflux medication use is more prevalent amongst extremely premature infants. Half of the infants born at $<28$ weeks' $G A$ received anti-reflux medication in hospital and a quarter at discharge. This group also received antireflux medications for, on average, 9 days more compared to the infants born at 28-31 weeks' GA.
A wide range of clinical signs/symptoms amongst preterm infants are linked to GORD but none are pathognomonic making it difficult to diagnose clinically. In this database, a diagnosis of GORD was reported in $16 \%$ of infants born at $<32$ weeks' GA. Previous estimates from UK survey data $^{10}$ report a higher prevalence at $22 \%$ in infants born at $<34$ weeks' GA. Such surveys, however, report the incidence as estimated by the responding clinician and therefore may not represent true prevalence. We found a lower prevalence reported in the NNRD among $<32$ weeks' GA infants while Jadcherla et al. ${ }^{3}$, in a study from 33 hospitals in the United States, reported that $10 \%$ of all preterm infants ( $\leq 37$ weeks GA) had a diagnosis of GORD. The information available in the NNRD does not include how the diagnosis was made and whether any investigations or diagnostic tools were used to determine the presence and severity of GORD. Investigations such as $\mathrm{pH}$ monitoring, multichannel intraoesophageal impedance monitoring, contrast fluoroscopy, endoscopy and biopsy are infrequently used for diagnosis in UK neonatal practice and most cases, are diagnosed based on clinical features or clinical features and a therapeutic trial with anti-reflux medications. ${ }^{10,11}$ Although $\mathrm{pH}$ monitoring is available in most neonatal units, less than a third report using it ${ }^{10}$ possibly because it may not be a reliable marker of GORD in preterm infants. The stomach $\mathrm{pH}$ of preterm infants is rarely $<4$ due to frequent milk feeding and a higher baseline $\mathrm{pH}^{7}$ Moreover, higher acidity in the 
oesophagus does not correlate with symptoms of GORD in newborn infants. ${ }^{13}$

Most infants had GORD diagnosis later in the course of their neonatal stay. The median (IQR) of PMA at first GORD diagnosis was 36 weeks (33-38). Difficulties with feeding such as vomiting, regurgitation or discomfort after large volume feeds often manifest only after the establishment of higher feed volumes. Airways are often protected with devices such as endotracheal tubes and breathing supported with additional respiratory support in the early days after birth. Cardio-respiratory events such as episodes of desaturations may manifest later. These signs may be taken to be suggestive of GOR and may prompt trials of treatment and diagnosis of GORD.

When comparing infants who had a recorded diagnosis of GORD with those who did not, we found that those with GORD diagnosis were more premature and lighter at birth. This is consistent with studies showing that the occurrence of GOR and GORD were higher in younger preterm infants as compared to more matured preterm infants, as well as in lower birth weight infants. ${ }^{3}$ This could be because mechanisms of GORD such as transient lower oesophageal sphincter relaxation (TLESR) are more frequent in more premature infants. ${ }^{13}$ TLESR can lower oesophageal sphincter pressure to levels at, or below, intragastric pressure causing regurgitation of stomach contents. In terms of physiological or structural maturity, less mature infants have lower oesophageal peristaltic velocity as well as a shorter oesophagus and lower oesophageal sphincter (LES) which would exacerbate GOR events more than in more mature infants. ${ }^{7}$ Other factors related to preterm infants' risk of GORD are relatively abundant milk intake, body position and cow's milk protein allergy. ${ }^{13}$ Nonpharmacological methods that address some of these can reduce the use of H2RA and PPIs. ${ }^{14}$

Distinguishing physiological GOR and pathological GORD is challenging. Symptoms related to GORD such as excessive crying, regurgitation/vomiting, irritability and back arching frequently occur in healthy infants, and there is also no evidence that these symptoms are temporally associated with GOR events. ${ }^{15}$ Similarly cardio-respiratory events often linked to GORD such as apnoea, episodes of oxygen desaturation and swallowing dysfunction are common in all preterm infants and studies show that suspected clinical reflux behaviours do not correlate with acid reflux as measured by oesophageal multichannel impedance. ${ }^{15}$

Interestingly, we found a higher prevalence of use of anti-reflux medication compared to the prevalence of GORD diagnosis. This suggests that anti-reflux medications are prescribed without the diagnosis of GORD such as for a trial of a therapy which may be used as a diagnostic tool. A survey of UK clinicians showed that $50 \%$ of the respondents regularly used clinical features plus therapeutic trials to determine the diagnosis of GORD. ${ }^{10}$ Trials of anti-reflux medications to establish a diagnosis of GORD are recommended in older infants and children ${ }^{1}$ but such therapeutic trials are not recommended in preterm infants because clinical signs and symptoms do not correlate with acidic or non-acidic reflux and the signs improve with time without treatment. ${ }^{7}$ Such empirical treatments can alter the gut microbiome enhancing pathogenic flora and increase the risks of NEC and infections. ${ }^{6}$ Shakeel et al. ${ }^{14}$ reported large reductions in the use of anti-reflux medications with the implementation of guidelines that recommended conservative and expectant management of GOR symptoms until preterm infants were 37 weeks PMA, demonstrating that standardised clinical practice guidelines, with appropriate education that addresses knowledge gaps in GORD management, can reduce inappropriate use of anti-reflux medications.

Although Gaviscon, a preparation of sodium alginate combined with sodium bicarbonate, was the most prescribed among the total cohort ( $57 \%$ of all infants $<32$ weeks' GA), H2RAs, mainly ranitidine, use was more prevalent among the extremely preterm infants, prescribed to $64 \%$. In addition, $20 \%$ received PPIs. These frequencies of use are higher, participially for H2RA use, than that reported from 43 US hospitals between 2006 and 2013 where, among infants $<29$ weeks' GA, $24 \%$ had any H2RA and $17 \%$ had any PPI. ${ }^{8}$

Not only did a larger proportion of extremely preterm infants receive anti-reflux medications, they also received them for longer. The median duration of anti-reflux prescription was 9 days longer for those born at $<28$ weeks' GA as compared to those born at 28-31 weeks' GA. The US study recorded median (IQR) treatment duration of $15(6-35)$ days but this included preterm infants of 26-36 weeks GA. ${ }^{8}$ The longer use of medications among extremely preterm infants could possibly be due to a more prolonged course of GORD due to greater immaturity or because they usually stayed longer in the neonatal unit which opens a longer window of opportunity for medication use as compared to those who were born at later gestations.

It was alarming to note the high use of prokinetics which was prescribed to $45 \%$ of the cohort considering that the warning issued by the Food and Drug Administration (FDA) in 2009 and by the European Medicines Agency (EMA) in $2013^{1}$ which clearly stated that their potential side effects counterweigh the possible benefits of these medications for the treatment of GORD in infants $<12$ months of age. ${ }^{16}$ It was, therefore, reassuring that the use has decreased significantly, particularly since 2013, perhaps in keeping with the release of the EMA statement and, later, the UK Medicine and Healthcare products Regulatory Authority (MHRA) announcement on the risk of adverse cardiac events with domperidone. ${ }^{1}$

The use of H2RAs and Gaviscon also declined after 2013, although not as rapidly. At this time, emerging evidence suggested that the use of H2RA is associated with higher risks of adverse effects including infections and $\mathrm{NEC}^{17,18}$, although this was already mentioned in earlier guidelines. ${ }^{16}$ Gaviscon, perhaps seen as more innocuous due to lack of evidence of such associations also continues to be used frequently. It can decrease the number of acidic GOR episodes, total oesophageal acid exposure and the frequency of regurgitation events ${ }^{19,20}$ but there is no evidence to show that it alleviates clinical signs and symptoms. ${ }^{19,21}$

The use of PPIs has increased more than twofold during the study period. A study conducted by Omari et al. ${ }^{22}$ showed that omeprazole, a PPI, is effective in reducing the frequency of acid reflux episodes and oesophageal acid exposure but has no impact on clinical signs/symptoms in preterm infants. Moore et al. ${ }^{23}$ showed that omeprazole reduced the reflux index as compared to placebo, but changes in clinical features were the same in both groups. These findings are similar to studies using other PPIs such as lansoprazole and esomeprazole. ${ }^{24,25}$

The use of the NNRD as source data has limitations as it is not used for primary prescribing, and diagnoses are not recorded in a standardised manner such as using ICD codes. There is a risk of data entry errors and missing data. The lower prevalence in NNRD data than the use of anti-reflux medications could also reflect diagnoses not recorded or may represent the lack of formal diagnosis. Additionally, due to the limited information included in the database, we are unable to investigate the details of these prescriptions including the dose and frequency of administration, adverse events, response to medications, and why they were stopped which are all vital information required to assess the rational use of any medication. However, NNRD is the only large database of neonatal data in the UK. The robustness and validity of NNRD data have been previously demonstrated and it is increasingly used for research. ${ }^{26}$ The use of this database allowed us to analyse data from over 58,000 infants born at $<32$ weeks' GA representing almost all the eligible population in England and Wales from 2010 to 2017. To our knowledge, this is the first and largest attempt in analysing the prevalence of GORD diagnosis and the prescriptions of anti-reflux medications in England and Wales with data from 200 neonatal units. The results provide 
valuable information on the use of anti-reflux medications in a large population over a long period of time.

GORD remains an area fraught with diagnostic and management conundrums ${ }^{27}$ and the high prevalence of use of medications despite the lack of evidence of benefit and associations with potential harm, reflects these uncertainties. While large randomised controlled trials are required to evaluate the benefits and harms of using anti-reflux medications in preterm infants, such studies cannot be performed without some clarity or consensus on how to identify infants who have pathological GORD. When perception of "troublesome" symptoms are used to diagnose and treat GORD, trials show no evidence of benefit. ${ }^{28}$ Objective determination of diagnosis such as using the acid reflux index ${ }^{27}$ can identify infants with true oesophageal acid exposure but do not correlate with clinical signs and symptoms. Additionally, these investigations are not used routinely in the UK ${ }^{10,11}$ and therefore findings from studies using such methods of diagnosis may not readily translate to change in practice. Pragmatic studies that investigate methods of identifying infants thought to have "troublesome" GORD, tool to monitor progression of signs and symptoms attributed to GOR, and evaluation of practices that could reduce the use of anti-reflux medications may help achieve rational treatment for preterm infants.

\section{CONCLUSION}

Our findings suggest that anti-reflux medications are frequently prescribed in very preterm infants despite evidence suggesting that they are not effective and may be harmful. Further research and clear guidelines for diagnosing GORD and for rationalising the pharmacological management of GORD are required.

\section{DATA AVAILABILITY}

Data are available from the corresponding author upon request.

\section{REFERENCES}

1. Rosen, R. et al. Pediatric gastroesophageal reflux clinical practice guidelines: joint recommendations of the North American Society for Pediatric Gastroenterology, Hepatology, and Nutrition and the European Society for Pediatric Gastroenterology, Hepatology, and Nutrition. J. Pediatr. Gastroenterol. Nutr. 66, 516-554 (2018).

2. The National Institute for Health and Care Excellence (NICE). Gastro-oesophageal Reflux Disease in Children and Young People: Diagnosis and Management (2019).

3. Jadcherla, S. R. et al. Practice variance, prevalence, and economic burden of premature infants diagnosed with GERD. Hosp. Pediatr. 3, 335-341 (2013).

4. BMJ Group, Pharmaceutical Press, and RCPCH Publications, London. Paediatric Formulary Committee. BNF for Children (online) http://www.medicinescomplete. com (2019).

5. Barney, C. K. et al. Lansoprazole, ranitidine, and metoclopramide: comparison of practice patterns at 4 level III NICUs within one healthcare system. Adv. Neonatal Care Off. J. Natl Assoc. Neonatal Nurses 9, 129-131 (2009).

6. Santos, V. S. et al. Association between histamine-2 receptor antagonists and adverse outcomes in neonates: a systematic review and meta-analysis. PLOS ONE 14, e0214135 (2019).

7. Eichenwald, E. C. Diagnosis and management of gastroesophageal reflux in preterm infants. Pediatrics 142, 11 (2018).

8. Slaughter, J. L., Stenger, M. R., Reagan, P. B. \& Jadcherla, S. R. Neonatal histamine2 receptor antagonist and proton pump inhibitor treatment at United States Children's Hospitals. J. Pediatr. 174, 63-70.e3 (2016).

9. Malcolm, W. F. et al. Use of medications for gastroesophageal reflux at discharge among extremely low birth weight infants. Pediatrics 121, 22-27 (2008).

10. Dhillon, A. \& Ewer, A. Diagnosis and management of gastro-oesophageal reflux in preterm infants in neonatal intensive care units. Acta Paediatr. 93, 88-93 (2007).

11. Rossor, T., Andradi, G., Bhat, R. \& Greenough, A. Investigation and management of gastro-oesophageal reflux in United Kingdom neonatal intensive care units. Acta Paediatr. 107, 48-51 (2018).

12. Gale, C. \& Morris, I. The UK National Neonatal Research Database: using neonatal data for research, quality improvement and more. Arch. Dis. Child.-Educ. Pract. Ed. 101, 216-218 (2016).
13. Gulati, I. K. \& Jadcherla, S. R. Gastroesophageal reflux disease in the neonatal intensive care unit infant: who needs to be treated and what approach is beneficial? Pediatr. Clin. North Am. 66, 461-473 (2019).

14. Shakeel, F. M. et al. Decreasing inappropriate use of antireflux medications by standardizing gastroesophageal reflux disease management in NICU. Pediatr. Qual. Saf. 6, e394 (2021).

15. Funderburk, A. et al. Temporal association between reflux-like behaviors and gastroesophageal reflux in preterm and term infants. J. Pediatr. Gastroenterol. Nutr. 62, 556-561 (2016).

16. Vandenplas, Y. et al. Pediatric gastroesophageal reflux clinical practice guidelines: Joint Recommendations of the North American Society for Pediatric Gastroenterology, Hepatology, and Nutrition (NASPGHAN) and the European Society for Pediatric Gastroenterology, Hepatology, and Nutrition (ESPGHAN). J. Pediatr. Gastroenterol. Nutr. 49, 498-547 (2009).

17. Terrin, G. et al. Ranitidine is associated with infections, necrotizing enterocolitis, and fatal outcome in newborns. Pediatrics 129, e40-e45 (2012).

18. Bilali, A., Galanis, P., Bartsocas, C., Sparos, L. \& Velonakis, E. H2-blocker therapy and incidence of necrotizing enterocolitis in preterm infants: a case-control study. Pediatr. Neonatol. 54, 141-142 (2013).

19. Corvaglia, L. et al. The efficacy of sodium alginate (Gaviscon) for the treatment of gastro-oesophageal reflux in preterm infants. Aliment Pharm. Ther. 33, 466-470 (2011).

20. Atasay, B., Erdeve, O., Arsan, S. \& Türmen, T. Effect of sodium alginate on acid gastroesophageal reflux disease in preterm infants: a pilot study. J. Clin. Pharmacol. 50, 1267-1272 (2010).

21. Kwok, T. C., Ojha, S. \& Dorling, J. Feed thickener for infants up to six months of age with gastro-oesophageal reflux. Cochrane Database Syst. Rev. 12, CD003211 (2017).

22. Omari, T. I., Haslam, R. R., Lundborg, P. \& Davidson, G. P. Effect of omeprazole on acid gastroesophageal reflux and gastric acidity in preterm infants with pathological acid reflux. J. Pediatr. Gastroenterol. Nutr. 44, 41-44 (2007).

23. Moore, D. J. et al. Double-blind placebo-controlled trial of omeprazole in irritable infants with gastroesophageal reflux. J. Pediatr. 143, 219-223 (2003).

24. Davidson, G. et al. Efficacy and safety of once-daily esomeprazole for the treatment of gastroesophageal reflux disease in neonatal patients. J. Pediatr. 163, 692-698.e2 (2013).

25. Orenstein, S. R., Hassall, E., Furmaga-Jablonska, W., Atkinson, S. \& Raanan, M. Multicenter, double-blind, randomized, placebo-controlled trial assessing the efficacy and safety of proton pump inhibitor lansoprazole in infants with symptoms of gastroesophageal reflux disease. J. Pediatr. 154, 514-520.e4 (2009).

26. Sand, L. et al. Observational cohort study of changing trends in non-invasive ventilation in very preterm infants and associations with clinical outcomes. Arch. Dis. Child. Fetal Neonatal Ed. https://doi.org/10.1136/archdischild-2021-322390 (2021).

27. Jadcherla, S. R. et al. Role of feeding strategy bundle with acid-suppressive therapy in infants with esophageal acid reflux exposure: a randomized controlled trial. Pediatr. Res. 89, 645-652 (2021).

28. Dermyshi, E., Mackie, C., Kigozi, P., Schoonakker, B. \& Dorling, J. Antacid therapy for gastroesophageal reflux in preterm infants: a systematic review. BMJ Paediatr. Open 2, e000287 (2018).

\section{ACKNOWLEDGEMENTS}

Electronic patient data recorded at participating neonatal units that collectively form the United Kingdom Neonatal Collaborative are transmitted to the Neonatal Data Analysis Unit to form the National Neonatal Research Database (NNRD). We are grateful to all the families that agreed to the inclusion of their baby's data in the NNRD, the health professionals who recorded data, and the Neonatal Data Analysis Unit team.

\section{AUTHOR CONTRIBUTIONS}

H.B.A.H., S.O., L.S. and H.B. conceptualised and designed the study and reviewed and revised the manuscript. H.B.A.H. performed the data analysis and drafted the initial manuscript. All authors approved the final manuscript as submitted and agree to be accountable for all aspects of the work.

\section{FUNDING INFORMATION}

H.B.A.H. is supported by a Ph.D. scholarship from the Ministry of Higher Education Malaysia and Universiti Kebangsaan Malaysia (UKM). 


\section{COMPETING INTERESTS}

The authors declare no competing interests.

\section{ETHICS APPROVAL}

Ethical approval was obtained from the Yorkshire \& The Humber-Leeds East Research Ethics Committee and Health and Care Research Wales (HCRW) (REC reference: 18/YH/0209; Date of approval: 25 May 2018).

\section{CONSENT TO PARTICIPATE}

Informed consent was not asked from individuals whose data were included in this study as the historical data used were held in the pre-existing, REC-approved NNRD. Data held in the NNRD are de-identified and no patient identifiers were used in this study.

\section{CONSENT FOR PUBLICATION}

Publication consent was not asked from individuals whose data were included in this study as the historical data used were held in the pre-existing, REC-approved NNRD. Data held in the NNRD are de-identified and no patient identifiers were used in this study.

\section{ADDITIONAL INFORMATION}

Correspondence and requests for materials should be addressed to Shalini Ojha.

Reprints and permission information is available at http://www.nature.com/ reprints

Publisher's note Springer Nature remains neutral with regard to jurisdictional claims in published maps and institutional affiliations.

(C) Open Access This article is licensed under a Creative Commons Attribution 4.0 International License, which permits use, sharing, adaptation, distribution and reproduction in any medium or format, as long as you give appropriate credit to the original author(s) and the source, provide a link to the Creative Commons license, and indicate if changes were made. The images or other third party material in this article are included in the article's Creative Commons license, unless indicated otherwise in a credit line to the material. If material is not included in the article's Creative Commons license and your intended use is not permitted by statutory regulation or exceeds the permitted use, you will need to obtain permission directly from the copyright holder. To view a copy of this license, visit http://creativecommons. org/licenses/by/4.0/.

(C) The Author(s) 2021 